\title{
Microimmersion lens LEDs for portable photoacoustic methane sensors
}

\author{
Boris Matveev $^{1}$, Maxim Remennyy ${ }^{1}$, Karandashev Sergey $^{1}$, Kimmo Keränen $^{2}$, \\ Heini Saloniemi ${ }^{2}$, Jyrki Ollila ${ }^{2}$, Tom Kuusela ${ }^{3}$, Ismo Kauppinen ${ }^{4}$ \\ ${ }^{1}$ loffe Institute, 26 Polytekhnicheskaya, St Petersburg 194021, RUSSIAN FEDERATION \\ ${ }^{2}$ VTT, Kaitoväylä 1, 90570 Oulu, FINLAND \\ ${ }^{3}$ University of Turku, Department of Physics and Astronomy, FI-20014 University of Turku, \\ FINLAND \\ ${ }^{4}$ Gasera Itd., Tykistökatu 4, 20520 Turku, FINLAND \\ Corresponding e-mail address: bmat@iropt3.ioffe.ru
}

\begin{abstract}
.
Microimmersion lens mid-infrared light emitting diodes (mid-IR LEDs) operating in the $3.4 \mu \mathrm{m}$ wavelength range, which covers the absorption bands of many important hydrocarbons $\left(\mathrm{CH}_{4}, \mathrm{C}_{3} \mathrm{H}_{8} \ldots\right)$, have been studied with respect to their application in cantilever enhanced photoacoustic trace gas detection technique
\end{abstract}

Key words: IR LEDs, photoacoustic methane sensors, NDIR spectroscopy, immersion lenses

\section{Introduction}

There is a wide range of applications in which sensitive trace gas detection is needed, and both non-spectroscopic and spectroscopic methods are used for these purposes. The photoacoustic (PA) gas detection can offer high sensitivities without excessive long absorption lengths since the sensitivity is limited by the pressure increment that does not change as the gas volumes are decreased.

The highest sensitivities have been achieved so far in PA systems equipped with micromachined silicon cantilever whose displacement is measured via a Michelson-type laser interferometer. Light emitting diodes (LEDs) can offer additional benefits since LED output is easily modulated by current pulsing with frequencies up to several $\mathrm{MHz}$, besides they have small size and power consumption,. Recently it has been reported that with immersion lens mid-IR LEDs and interferometric cantilever microphone the ppmlevel gas detection limits can be achieved [1]. The above LEDs were equipped with $3.5 \mathrm{~mm}$ wide lenses that were 10 -fold wider than the LED active area of appr. $250 \times 250 \mu \mathrm{m}$ dimensions. Large lens dimensions provides both good outcoupling efficiency and imaging of the active area at a distance of $\sim 30 \mathrm{~mm}$ from the LED [']. However the image dimensions were increased with respect to the original and thus it was not possible to utilize properly all LED output power - well known problem in many instruments with entrance slid or detector elements of limited dimensions, e.g. in the PA analyzers with miniature gas cell and coherently with small entrance windows.

Formation of a chalcogenide glass drop directly onto a LED surface enable efficient radiation outcoupling from a LED since the above glass possess both high transparency and high refractive index $(n=2.3-2.5)[2,4]$. The above features together with high surface tension and good adhesion to semiconductor make chalcogenide immersion lens $\mathrm{T}$ The above features together with high surface tension and good adhesion to semiconductor make chalcogenide immersion lens technology attractive for analyzers with miniature gas cells and entrance windows.

In this paper we study microimmersion lens LEDs that to our understanding solve the problem, that is, provide both high extraction and collection efficiency in miniature optical systems, based on mid-IR LED technology.

\section{Experimental details}

Single heterostructures were grown by the LPE method and consisted of $350 \mu \mathrm{m}$ thick heavily doped $n^{+}-\ln A s(\mathrm{Sn})$ substrates with an electron concentration $\mathrm{n}=(3 \div 6) \cdot 10^{18} \mathrm{~cm}^{-3}$ and two epilayers. They represented $2 \div 4 \mu \mathrm{m}$ thick $\mathrm{n}$ InAs active region and 2-3 $\mu \mathrm{m}$ thick wide-gap $p$ $\ln A s_{1-x-y} S b_{x} P_{y}(Z n)\left(x \approx 0.09, y \approx 0.18, p=2 \div 510^{17}\right.$ $\mathrm{cm}^{-3}$ ) cap layer. The obtained heterostructures were processed by a wet photolithography into $\sim 400 \times 590 \mu \mathrm{m}$ chips with $\sim 170 \times 170 \mu \mathrm{m}$ mesas with mesa depth up to $25-30 \mu \mathrm{m}$, contact areas 
being on the same (epitaxial) chip side. Both chip contacts were connected to contact areas of a $1.8 \times 0.9 \times 0.4 \mathrm{~mm}$ semiinsulating Sisubmount by soldering at $180^{\circ} \mathrm{C}$. Finally the Si submounts with LED chips were soldered onto a TO-18. Chalcogenide glass lens was formed directly onto free LED surface (InAs substrate) as shown in Fig.1.

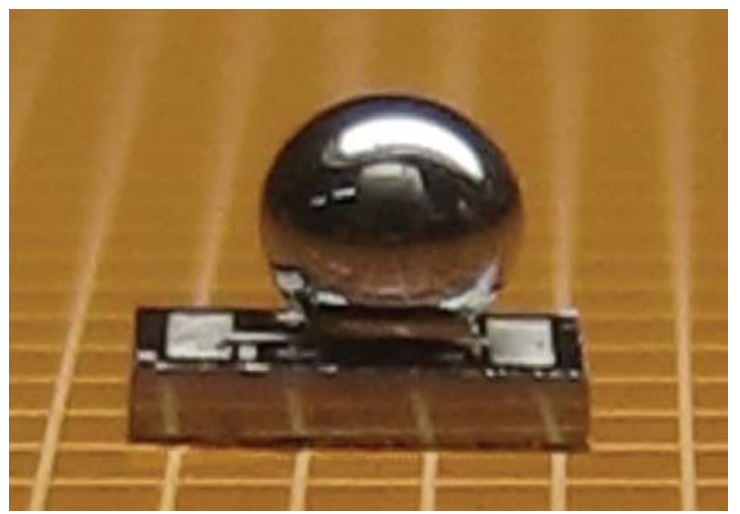

Fig. 1. Photo of microimmersion lens LED onto $1.8 \times 0.9 \times 0.4 \mathrm{~mm} \mathrm{Si}$ submount. Grid cells are of $0.5 \times 0.5 \mathrm{~mm}$ dimensions.

$X-Y$ movable stage was used for distribution of radiation along the plane with area up to $20 \times 20$ $\mathrm{mm}$ placed at distance $L$ of 0,5 and $10 \mathrm{~mm}$ from the LED (see Fig.2). Home made InAs flip chip photodiode (PD) with $210 \times 210 \mu \mathrm{m}$ active area was used for intensity registration performed at LED pulsing at $500 \mathrm{~Hz}$ with 1:1 duty cycle). "IR images" or intensity "maps" consisted of $40 \times 40$ (step $500 \mu \mathrm{m}$ ) pixels, no corrections accounted for variation of incident angle $\theta$ have been made.

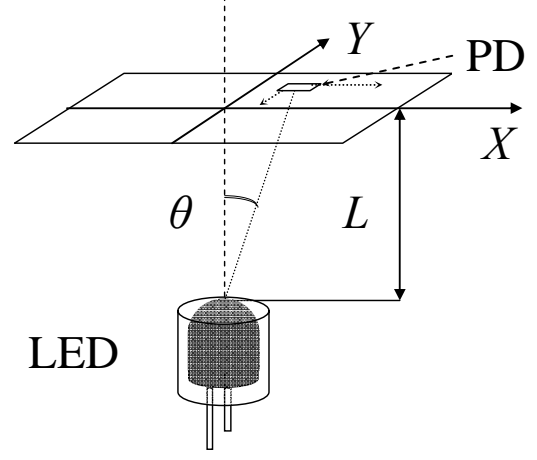

Fig. 2. Schematic of the far field measurements with the $\mathrm{X}-\mathrm{Y}$ scanning system

\section{Results and discussion}

Fig.3 presents dependence of relative output power on lens dimensions (lens pedestal height $\mathrm{H}$ ) in microimmersion lens LEDs with a fixed value of surface radii (lateral dimensions) - 1.1 $\mathrm{mm}$ that is currently though to be optimal for applications in mind. It follows from Fig. 3 that the highest extraction efficiency (best power) is achieved at zero pedestal height $\mathrm{H}$ that corresponds to a hemispherical lens where all rays coming from the active area "hit" surface at nearly zero incident angle.

Unfortunately hemispherical lens provides the worst beam divergence and thus the collection efficiency for a concave concentrator optical scheme falls down to a minimum as seen from Fig.4.

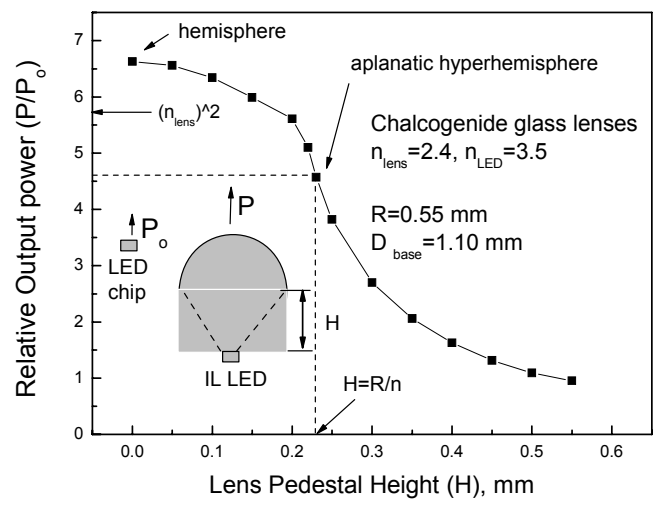

Fig. 3. Dependence of relative output power on lens dimensions (pedestal height $H$ ). $P_{0}$ and $P$ are the output power of plane chip and chip immersed into a chalcogenide glass lens respectively.

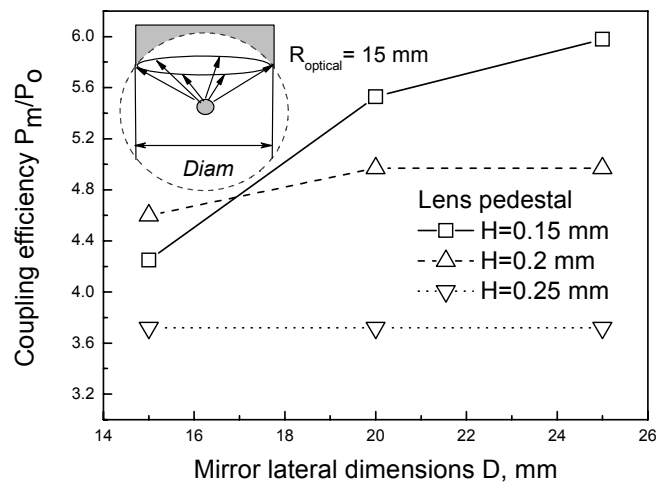

Fig. 4. Optical coupling efficiency for microimmersion lens $L E D$ - spherical mirror pair vs mirror diameter for mirror radii fixed at $15 \mathrm{~mm}$.

This is not a surprise that the increase of the pedestal height $\mathrm{H}$ is followed by a collection efficiency enhancement (see Fig.4) as lens is attaining ability to concentrate radiation into a spatially narrow beam with limited divergence. However, the extraction efficiency (Fig.3) is not $100 \%$ for the case as only limited number of incident angles are smaller than the angle of total internal reflection $(\theta$ cr. $=24.5$ deg. $)$. Conditions that provide both adequate outcoupling and coupling efficiency are thought to be such that $H=R / n$, where $n$ - is the lens refractive index $(n=2.4)$. These "aplanatic hyperhemisphere" lenses with $\mathrm{H}=\mathrm{R} / \mathrm{n}$ are well known in photodiode $[4,5,6]$ and $\operatorname{LED}[2,4,7]$ 
technology using $\mathrm{Si}$ or Ge lenses. In our case the condition $\mathrm{H}=\mathrm{R} / \mathrm{n}$ was hardly acheived as lens pedestal depends on radii (or lens mass). Nevertheless we were able to fabricate nearly identical immersion lens LED with quite nice average power of $120 \mu \mathrm{W}$ (at $100 \mathrm{~mA}$ ) and typical dependence of beam amplitude, FWHM presented in Fig.5. FWHM, $\beta$ and amplitudes (A) listed in Fig.5 were calculated from Gauss function approximation of the experimental data (line scan from beam center). As seen from Fig.5 microimmersion lens LED beam FWHM linearly increases as the distance $L$ is increased, the FWHM magnitude is smaller than expected for the plain LED chip. The latter "Lambertian" LED (A $\left.\left(\mathrm{L} / \operatorname{sqrt}\left(\mathrm{L}^{\wedge} 2+(\mathrm{x})^{\wedge} 2\right)\right)^{\wedge} 4\right)$ provides $\mathrm{FWHM}_{\mathrm{Lamb}}=13 \mathrm{~mm}$ against $\mathrm{FWHM}=$ $3.7 \mathrm{~mm}$ for microimmersion LEDs at $\mathrm{L}=10 \mathrm{~mm}$.

The collimating effect in our LEDs and high coupling efficiency in mirror based optical schemes are evident - there is minor change of the $A^{*} \beta^{\wedge} 2$ parameter accounting for the integral power that can be collected at a distance $\mathrm{L}$. The latter was confirmed by the Zemax analysis of the LED beams collected by spherical mirror and by experiments. Nearly $74 \%$ collection efficiency was achieved when LED was placed at a distance of $12 \mathrm{~mm}$ from gold plated mirror whose surface radius was $12.0 \mathrm{~mm}$ (the focus length $6.0 \mathrm{~mm}$ ), the diameter $12.0 \mathrm{~mm}$.

\section{Acknowledgements}

The work was supported in part by Seventh Frame Program of EC (FP7, № 224625 (MINIGAS)).

\section{References}

[1] T. Kuusela, J. Peura, B. A. Matveev , M. A. Remennyy, N. M. Stus', Photoacoustic gas detection using a cantilever microphone and III-V mid-IR LEDs, Vibrational Spectroscopy, 51(2), 289-293 doi.org/10.1016/j.vibspec.2009.08.001,

(2009),

[2] .M. N. V. Zotova, S. A. Karandashev, B. A. Matveev, M. A. Remennyi and N. M. Stus', "Radiation distribution in $3.4 \mu \mathrm{m}$ immersion lens LEDs in far field", Journal of Optical Technology, (2012) (in press)

[3] Chey J.W., Sultan P., Gerritsen H.J., Resonant photoacoustic detection of methane in nitrogen using a room temperature infrared light emitting diode, Appl.Optics, 26, 3192-3194 (1987)

[4] Boris A.Matveev, "LED-Photodiode Opto-pairs" in Mid-infrared Semiconductor Optoelectronics, Springer Series in OPTICAL SCIENCE, ISSN 0342-4111, 395- 428 (2006), doi: 10.1007/184628-209-8_12

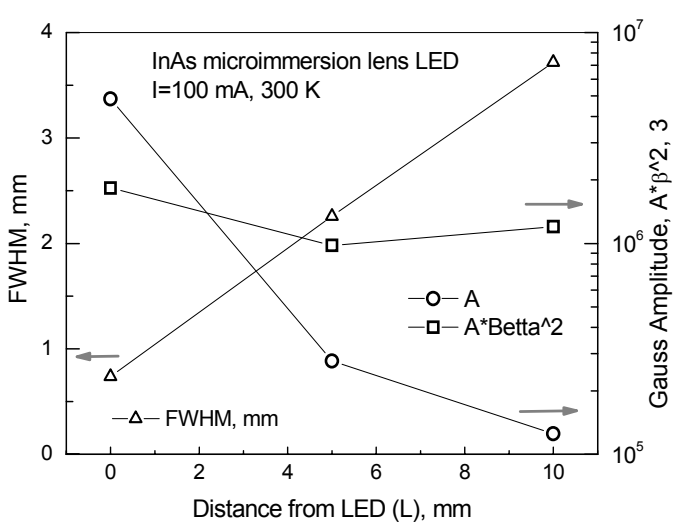

Fig. 5. Beam characteristics vs distance from $L E D$

\section{Conclusions}

Microimmersion lens technology provides several benefits for the optical instrumentation based on mid-IR components including small emitting size and ourtput power compared to conventional LED with Si lenses [8]. Low cost, small size of the miroimmerison lens LED are key features for portable instruments, e.g. based on PA spectroscopy. In near future experimental verification of applicability of the above technology to PA gas analyzer incorporating micromachined silicon cantilever whose displacement is measured via a novel laser interferometer will be made.

[5] R.C. Jones, Immersed radiation detectors, Appl.Optics, 1, 607-613 (1962)

[6] S.A. Karandashev, B.A. Matveev, M.A. Remennyi, A.A. Shlenskii, L.S. Lunin, V.I. Ratushnyi, A.V. Koryuk, N.G. Tarakanova,, Properties of GalnAsSb/GaSb (Lambda $=1.8-2.3$ um) Immersion Lens Photodiodes at 20-140 C, Semiconductors, 41 (11), 1369-1374 (2007)

[7] M. Aidaraliev, N. V. Zotova, S. A. Karandashev, B. A. Matveev, M. A. Remennyi, N. M. Stus', and G. N. Talalakin, Optically Pumped "ImmersionLens" Infrared Light Emitting Diodes Based on Narrow-Gap III-V Semiconductors, Semiconductors, 36 (7), 828-831(2002)

[8] www.mirdog.spb.ru 